\title{
A SEPARABLE NORMAL NONPARACOMPACT SPACE
}

\section{MARY ELLEN RUDIN}

A topological space $X$ is said to be paracompact [1] if for every open covering $G$ of $X$ there is a locally finite open covering $G^{\prime}$ of $X$ which is a refinement of $G$. ( $G^{\prime}$ is locally finite if every point of $X$ has a neighborhood which intersects only a finite number of members of $G^{\prime}$.) It is known that every paracompact Hausdorff space is normal [1] and that every metrizable space is paracompact [2]. Since every normal Hausdorff space with a countable base is metrizable, therefore, every normal Hausdorff space with a countable base is paracompact.

The purpose of this paper is to show that the existence of a countable base cannot be replaced by separability in this last statement.

THEOREM. There exists a separable normal Hausdorff space $X$ which is not paracompact and does not have the Lindelöf property.

In this paper Greek letters will always denote countable ordinals; and the letters $i, j, k, m$, and $n$ will stand for positive integers.

Construction of $X$. Let $A$ denote the set of all ordered pairs $(m, n)$ and $B$ the set of all countable ordinals. Then $P$ will be a point of $X$ if and only if $P$ is a term of $A$ or $B$.

For each $\alpha$, let $f_{\alpha}$ be a function defined on the positive integers, with values in the positive integers, such that: if $\alpha<\beta$, there exists an integer $m(\alpha, \beta)=m(\beta, \alpha)$ such that $f_{\alpha}(i)<f_{\beta}(i)$ whenever $i>m(\alpha, \beta)$. These functions are used to define the topology of $X$.

The set $N$ will be a neighborhood if and only if it belongs to one of the following classes.

(1) Every point of $A$ is a neighborhood of itself.

(2) If $\alpha$ is not a limit ordinal, then corresponding to each $n$ there is a neighborhood of $\alpha$ which consists of (a) $\alpha$ itself, and (b) all pairs $\left(k, f_{\alpha}(k)\right)$ with $k>n$.

(3) If $\alpha$ is a limit ordinal, choose a $\beta<\alpha$, and an $n(\gamma)$ for each $\gamma$ such that $\beta<\gamma \leqq \alpha$. For each such collection of choices there is a neighborhood of $\alpha$ which consists of:

(a) all $\gamma$ such that $\beta<\gamma \leqq \alpha$, and

(b) all pairs $\left(k, f_{\gamma}(k)\right)$ with $k>n(\gamma)$ and $\beta<\gamma \leqq \alpha$.

This completes the construction of $X$.

Since $A$ is dense in $X, X$ is separable; and since any two distinct points have disjoint neighborhoods, $X$ is a Hausdorff space.

Presented to the Society, August 13, 1955; received by the editors May 19, 1955. 
Proof that $X$ is NoRmal. Let $H$ and $K$ be closed and disjoint subsets of $X$. If both $H$ and $K$ are uncountable, then $H \cdot B$ and $K \cdot B$ are both uncountable, and there exist sequences $\beta_{1}, \beta_{2}, \beta_{3}, \cdots$ and $\alpha_{1}, \alpha_{2}, \alpha_{3}, \cdots$ such that for each $n, \beta_{n}$ belongs to $H, \alpha_{n}$ belongs to $K$, and $\beta_{n}<\alpha_{n}<\beta_{n+1}$. The common limit ordinal is a limit point of both $H$ and $K$; but $H$ and $K$ have no common limit point.

Hence at least one of these sets, say $H$, is countable. Choose $\alpha_{0}$ such that, if $\alpha$ is in $H, \alpha<\alpha_{0}$. The construction of disjoint open sets covering $H$ and $K$ will be carried out with the aid of the integers $n(\alpha)$ now to be defined for each $\alpha$.

I. For $\alpha>\alpha_{0}$, choose $n(\alpha)>m\left(\alpha_{0}, \alpha\right)$.

II. Order the ordinals which do not exceed $\alpha_{0}$ in a simple countable sequence $\alpha_{0}, \alpha_{1}, \alpha_{2}, \cdots$. Take $n\left(\alpha_{0}\right)=1$. Then having chosen $n\left(\alpha_{0}\right), \cdots, n\left(\alpha_{i-1}\right)$, choose $n\left(\alpha_{i}\right)>m\left(\alpha_{i}, \alpha_{j}\right)$ where $0 \leqq j<i$.

Suppose that $\alpha$ belongs to $K$. There exists an ordinal $\beta$ which is maximal with respect to two properties: (a) $\beta$ is in $H$, and (b) $\beta<\alpha$. (It is assumed here that $H$ contains ordinals less than $\alpha$; in the contrary case take $\beta=1$.) If $\alpha$ is a limit ordinal construct the neighborhood $U^{\prime}(\alpha)$ in accordance with (3) using the $\beta$ above and the $n(\gamma)$ 's described in I and II. If $\alpha$ is not a limit ordinal construct the neighborhood $U^{\prime}(\alpha)$ in accordance with (2), taking $n=n(\alpha)$. In either case, let $U(\alpha)$ be a neighborhood of $\alpha$ which does not intersect $H \cdot A$ such that $U(\alpha)$ is a subset of $U^{\prime}(\alpha)$.

If $\alpha$ is in $H$, carry out the same construction interchanging $H$ and $K$ to obtain a neighborhood $V(\alpha)$. Let $U$ and $V$ be the sum of all neighborhoods $U(\alpha)$ and $V(\alpha)$ for $\alpha$ in $K$ and $H$, respectively.

Let $U^{\prime}=U+(K \cdot A)$ and $V^{\prime}=V+(H \cdot A)$. Then $U^{\prime}$ and $V^{\prime}$ are open and disjoint coverings of $K$ and $H$, respectively.

Proof ThAT $X$ is NOT PARACOMPACT. Let $G$ be the set of all neighborhoods defined in (1), (2), and (3). Note that every member of $G$ is countable. If $G^{\prime}$ is any refinement of $G$ covering $X$, some term of $A$ is in uncountably many members of $G^{\prime}$. Hence $X$ is not paracompact.

The same covering $G$ shows that $X$ does not have the Lindelof property, since no countable subcollection of $G$ can cover $X$.

\section{REFERENCES}

1. J. Dieudonné, Une generalization des espaces compactes, J. Math. Pures Appl. (9) vol. 23 (1944) pp. 65-76.

2. A. H. Stone, Paracompactness and product spaces, Bull. Amer. Math. Soc. vol. 54 (1948) pp. 977-982.

\section{UNIVERSITY OF ROCHESTER}

